\title{
XVI. Notes on a modification of Maxwell's electrical theory
}

\section{Alex McAulay M.A.}

To cite this article: Alex McAulay M.A. (1893) XVI. Notes on a modification of Maxwell's electrical theory, Philosophical Magazine Series 5, 36:219, 175-188, DOI: 10.1080/14786449308620464

To link to this article: http://dx.doi.org/10.1080/14786449308620464

曲 Published online: 08 May 2009.

Submit your article to this journal $[\pi$

Џ Article views: 5

Q View related articles $₫$ 
burner" with a light-giving power of 33 candles, on the other side a standard candle. As the candle was gradually brought nearer the rotating photometer, the illumination and hues of the portions $a$ and $b$ (fig. 17) of the photometric ring approached equality, and when the candle was at a certain distance the difference of illumination and hues of $a$ and $b$ become indistinguishable, and only a band with a breadth of $a+b$ with a uniform surface was observed on the sides of the photometric ring.

The measures of the intensity of the light of the Welsbach lamp in terms of the standard candle were made by a friend. He made sixteen measures with my Rotating-disk Photometer and sixteen with the Bunsen Photometer.

The mean of the series of measures made with the Rotating-disk Photometer differed from the mean of the departure of the maximum and minimum measures from the mean by 1.49 per cent. of the mean intensity of the light of the Welsbach lamp.

The mean of the series of measures made with the Bunsen Photometer differed from the mean of the departure of the maxinum and minimum

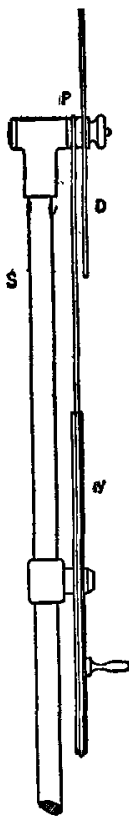

Fig.l8. measures from the mean by 5.22 per cent. of the mean intensity of the light of the Welsbach lamp.

Hoboken, N. J., U.S.A., April 15, 1893.

XVI. Notes on a Modification of Maxwell's Electrical Theory. By Alex. McAulay, M.A., University of Tasmania *.

1. TN the Phil. Trans., 1892, p. 685, I have developed a 1 modification of Maxwell's electrical theory. As frequent mention of this paper will be made below, it will be referred to briefly as 'Electromag.' The principal object of the paper was to free Maxwell's theory from certain difficulties I felt in applying it to gross matter, but I was careful to point out at the beginning that the theory developed might require serious modification for the æther. The principal object of the present paper is briefly to consider what modifications, if any, are required.

2. We shall require a proposition analogous to Prop. I. * Communicated by the Author. 
(p. 690) of 'Electromag.' For brevity I must refrain from redescribing my methods and notation and must constantly refer the reader to the original paper.

Prop. If $\tau_{a}, \tau_{b}$ be fluxes, $\mathrm{V} \tau_{a} \tau_{b} / \mathrm{D}$ is an intensity, $\mathrm{D}, \mathrm{D}^{\prime}$ being the densities of matter in the standard and present positions respectively. [In the original paper $\mathrm{D}$ was used for electrical density and $\mathrm{D}_{m}$ for material density.] $\mathrm{By}$ this is meant that $V \tau_{a}^{\prime} \tau_{b}^{\prime} / D^{\prime}$ bears the same relation to $\nabla \tau_{a} \tau_{b} / D$ as does $\sigma$ ' to $\sigma$ in equations (5) $\$ 7$ of 'Electromag.'

To prove, we have

Hence

$$
\tau_{a}^{\prime}=m^{-1} \chi \tau_{a}, \quad \tau_{b}^{\prime}=m^{-1} \chi \tau_{b}, \quad \mathrm{D}^{\prime}=m^{-1} \mathrm{D} .
$$

$$
\mathrm{V} \tau_{a}^{\prime} \tau_{b}^{\prime} / \mathrm{D}^{\prime}=m^{-1} \mathrm{~V} \chi \tau_{a} \chi \tau_{b} / \mathrm{D}=\chi^{\prime-1} \mathrm{~V} \tau_{a} \tau_{b} / \mathrm{D} \text {. }
$$

3. It may be noticed that the investigation in 'Electromag.' is not apparently complete for a perfect dielectric, though in a sense the equations are correct. For such a dielectric $x$ is zero, and it would seem to follow from equation (28) $\S 50$ that since $e=0, \mathrm{~d} \nabla l=0$. This, however, is not true, for in this case we have the condition $S \nabla d=0$, which introduces a new term into equation (28). It is easy to see, however, that the case is met by ignoring equation (28) $\$ 50$ and putting $\mathbf{E}$ of equation (29) equal to zero ; for $\mathbf{D}$ and d have now the same meaning, and account has been taken in equation (29) of $S \nabla D=0$. Since in the present paper $E$ of equation (29) is always zero; we will use $E$ to stand for the $\mathbf{E}_{0}$ of $\S 61$ (except that it is best to define it not as $\mathrm{RK}$ but as $-\mathrm{d} \nabla l$, or, what is the same in the present case, ${ }_{\mathrm{D}} \mathbf{D}()$ ). Thus $\mathbf{E}^{\prime}$ will have the meaning usually given to $\mathbf{E}$.

4. For a fluid we have for the $l^{\prime}$ of $\S 51$,

$$
l^{\prime}=-\mathrm{D}^{\prime}\left(\dot{\rho}^{\prime 2} / 2+w\right)+\left(2 \pi \mathrm{K}^{\prime-1} \mathbf{D}^{\prime 2}-\mu^{\prime} \mathbf{H}^{\prime 2} / 8 \pi\right), .
$$

where $w$ is the ordinary potential energy per unit mass due to compression or rarefaction, and is therefore a function of $\mathrm{D}^{\prime}$ only; and where $\mathrm{K}^{\prime}, \mu^{\prime}$ are scalars, which also can only be functions of $\mathrm{D}^{\prime}$.

Thus

$$
\begin{aligned}
& \alpha l^{\prime}=\alpha^{\prime} \cdot \partial l^{\prime} / \partial \mathrm{D}^{\prime}=\alpha\left(m^{-1}\right) \mathrm{D} \cdot \partial l^{\prime} \partial \mathrm{D}^{\prime} \\
& =-\frac{1}{2} \Psi^{-1} \mathrm{D}^{\prime} \partial l^{\prime} / \partial \mathrm{D}^{\prime}
\end{aligned}
$$

['Electromag.' § 54, equation (10)]. Hence [ibid. §54, equation (20)],

or [ibid. equation (21)]

$$
\left[\phi^{\prime}\right]=\mathrm{D}^{\prime} \partial l / \partial \mathrm{D}^{\prime}
$$

$$
\phi^{\prime}=-\left(p+p_{e}\right)-2 \pi K^{\prime-1} \mathbf{D}^{\prime}() \mathbf{D}^{\prime}-\mu^{\prime} \mathbf{H}^{\prime}() \mathbf{H}^{\prime} / 8 \pi, .
$$


where $p$ is put for the ordinary pressure given by

$$
p=\left(\mathrm{D}^{\prime} \partial / \partial \mathrm{D}^{\prime}-1\right)\left(\mathrm{D}^{\prime} w\right)=\mathrm{D}^{\prime 2} \partial w / \partial \mathrm{D}^{\prime}
$$

and $p_{e}$ is an additional pressure due to the variation of $\mathrm{K}^{\prime}$ and $\boldsymbol{\mu}^{\prime}$ with $\mathrm{D}^{\prime}$ given by

$$
p_{e}=\mathrm{D}^{\prime} \partial / \partial \mathrm{D}^{\prime} .\left(-2 \pi \mathrm{K}^{\prime-1} \mathrm{D}^{\prime 2}+\mu^{\prime} \mathrm{H}^{\prime 2} / 8 \pi\right) . .
$$

Now in equation (26), $\S 50$, put

Thus

$$
\mathbf{F}=\Phi_{f}^{\prime}=\Phi^{\prime}=0 \text {. }
$$

$$
\begin{aligned}
\mathrm{D}^{\prime} \bar{\rho}^{\prime} & =-\mathrm{D}^{\prime} \nabla^{\prime} \mathrm{W}+\phi^{\prime} \Delta^{\prime} \\
& =-\mathrm{D}^{\prime} \nabla^{\prime} \mathrm{W}-\nabla^{\prime}\left(p+p_{e}\right)-\frac{1}{2}\left(\mathrm{D}^{\prime} \Delta^{\prime} \mathrm{E}^{\prime}+\mathrm{B}^{\prime} \Delta^{\prime} \mathrm{H}^{\prime} / 4 \pi\right),
\end{aligned}
$$

where we have substituted $\mathbf{E}^{\prime}$ for $4 \pi \mathrm{K}^{\prime-1} \mathbf{D}^{\prime}$ and $\mathbf{B}^{\prime}$ for $\mu^{\prime} \mathbf{H}^{\prime}$. From these connexions and the equations

$$
\mathrm{S} \nabla^{\prime} \mathbf{B}^{\prime}=\mathrm{S} \nabla^{\prime} \mathbf{D}^{\prime}=0 \text {. . . . . . }
$$

it is quite easy to prove that

$$
\begin{aligned}
& \mathbf{B}^{\prime} \Delta^{\prime} \mathbf{H}^{\prime}=2 V B^{\prime} V \nabla^{\prime} \mathbf{H}^{\prime}-\mathbf{H}^{\prime 2} \nabla^{\prime} \mu^{\prime}, \\
& \mathbf{D}^{\prime} \Delta^{\prime} \mathbf{E}^{\prime}=2 V \mathbf{D}^{\prime} V \nabla^{\prime} \mathbf{E}^{\prime}-\mathbf{E}^{\prime 2} \nabla^{\prime} K^{\prime} / 4 \pi,
\end{aligned}
$$

and therefore that

$$
\begin{aligned}
& -\nabla^{\prime} p_{e}-\frac{1}{2}\left(\mathbf{D}^{\prime} \Delta^{\prime} \mathbf{E}^{\prime}+\mathbf{B}^{\prime} \Delta^{\prime} \mathbf{H}^{\prime} / 4 \pi\right) \\
& \quad=-V\left(\mathbf{D}^{\prime} V \nabla^{\prime} \mathbf{E}^{\prime}+\mathbf{B}^{\prime} V \nabla^{\prime} \mathbf{H}^{\prime} / 4 \pi\right)-\mathrm{D}^{\prime} \nabla^{\prime} \mathrm{P}_{e},
\end{aligned}
$$

where

$$
8 \pi \mathrm{P}_{e}=\mathrm{E}^{\prime 2} \partial \mathrm{K}^{\prime} / \partial \mathrm{D}^{t}+\mathrm{H}^{\prime 2} \partial \mu^{\prime} \partial \mathrm{D}^{\prime} . . .
$$

Hence, putting as usual

we obtain

$$
\mathrm{P}=\int\left(d p / \mathrm{D}^{\prime}\right), \ldots . \quad \cdot \quad \cdot \quad .
$$

$$
\ddot{\rho}^{\prime}=-\nabla^{\prime}\left(\mathrm{W}+\mathrm{P}+\mathrm{P}_{e}\right)-\mathrm{V}\left(\mathbf{D}^{\prime} \mathrm{V} \nabla^{\prime} \mathbf{E}^{\prime}+\mathbf{B}^{\prime} \mathrm{V} \nabla^{\prime} \mathbf{H}^{\prime} / 4 \pi\right) / \mathrm{D}^{\prime}, .
$$

which, when the electrical terms are omitted, is the usual hydrodynamical equation of motion.

Put now

$$
\dot{\rho}^{\prime}=\sigma^{\prime}, \quad . \quad \cdot \quad . \quad . \quad \cdot .
$$

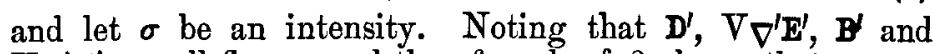
$\mathrm{V} \nabla^{\prime} \mathbf{H}^{\prime}$ are all fluxes, and therefore, by $\S 2$ above, that

$$
\mathrm{V}\left(\mathbf{D}^{\prime} \mathrm{V} \nabla^{\prime} \mathbf{E}^{\prime}+\mathbf{B}^{\prime} V \nabla^{\prime} \mathbf{H}^{\prime} / 4 \pi\right) / \mathbf{D}^{\prime}
$$

is an intensity, we obtain from equation (8)

$$
\chi^{\prime} \dot{\sigma}^{\prime}=-\nabla\left(\mathrm{W}+\mathrm{P}+\mathrm{P}_{e}\right)-\mathrm{V}(\mathbf{D V} \nabla \mathbf{E}+\mathbf{B V} \nabla \mathbf{H} / 4 \pi) / \mathrm{D} .
$$

Now

$$
\chi^{\prime} \dot{\sigma}^{\prime}=d\left(\chi^{\prime} \sigma^{\prime}\right) / d t-\dot{\chi}^{\prime} \sigma^{\prime}=\dot{\sigma}+\nabla_{1} S \sigma_{1}^{\prime} \sigma^{\prime} .
$$


Also

$$
\nabla_{\nabla} \mathbf{H}=4 \pi \dot{\mathbf{D}}, \quad V_{\nabla} \mathbf{E}=-\dot{\mathbf{B}} ; . . .
$$

the first being given by equations (36) and (37) of $\$ 50$ of 'Electromag.', and the second by equation $(20) \S 62$, and equation (34) $\$ 50$. Thus

$$
d(\sigma-\mathrm{VDB} / \mathrm{D}) / d t=-\nabla\left(\mathrm{W}+\mathrm{P}+\mathrm{P}_{\epsilon}+\sigma^{12} / 2\right) .
$$

5. The intensity VDB/D I shall call the Poynting intensity. For the present position of matter it is parallel to the Poynting flux $\mathrm{VE}^{\prime} \mathbf{H}^{\prime} / 4 \pi$. In fact, $\mathbf{P}$ being the Poynting flux and $\mathbf{Q}$ the Poynting intensity,

$$
\mathrm{D}^{\prime} \mathbf{Q}^{\prime}=v^{-2} \mathbf{P}^{\prime}, \quad \text {. . . . . . . . }
$$

where $v$ is the velocity $1 / \nu\left(K^{\prime} \mu^{\prime}\right)$ of light in the dielectric. [Note that $\mathbf{P}$ here is not quite the same as the $\mathbf{P}$ of $\$ 103$ of 'Electromag.', as that includes $\left.-\phi \chi^{\prime} \dot{\rho}^{\prime}=-\phi \sigma.\right]$

It will thus be seen that the term $V \mathbf{D B} / \mathrm{D}$ is in all ordinary dielectrics utterly insignificant compared with the term $\sigma$ in equation (12), and probably it would be quite hopeless to detect it by experiment, especially as all fluids are far from perfect.

Operating on equation (12) by V. $\nabla$ we see that the flux $\nabla_{\nabla}(\sigma-Q)$ is a constant for every element of the dielectric. Thus Helmholtz's theorems concerning vortices must be modified. by substituting the velocity - the Poynting intensity for the velocity. This may be obtained in a different way. Take the line-integral of both sides of eq. (12) between two particles of the fluid. We find that Lord Kelvin's theorem concerning flow must be modified by making the same change and further adding $\mathrm{P}_{e}$ to the expression $\mathrm{W}+\mathrm{P}+\sigma^{2} / 2$, which occurs in that theorem.

6. Though the effect of the Poynting intensity appearing in the equation of motion is insignificant in its influence on the motion of gross matter, it may be quite otherwise in vacuo. It was, in fact, by considering the case of the rether that I was led to eq. (12).

Turning now to the æther, we will consider three cases:-

I. The one mentioned in $\$ 2$ of 'Electromag.,' viz., where the density $\mathrm{D}$ is zero and the ordinary potential energy $w$ is also zero.

II. Where D is small (compared with the density of gross matter) but not zero, and where $w$ is still zero.

III. Where $\mathrm{D}$ has any value not large and where $w$ (no longer assumed merely a function of $D^{\prime}$ ) varies largely with strain except in the neighbourhood of gross matter.

7. Case I. is an extreme one, but seems to me the most natural to make on the theory developed in 'Electromag.' 
Before considering this case a word or two of general import will be given.

I cannot see that Maxwell has shown his right to ignore, as he does in considering the propagation of waves, the equation of motion of the medium. It is not hard to show that we may disregard it if we accept his equations as literally true for rapid waves in a medium of ordinary density. But may we do so for the æther, and may we do so in the case of gases where the gross matter present has practically no effect on electrical actions?

By ignoring the equation Maxwell is led to the conclusion that the rther when it is excited by electromagnetic waves exerts a pressure on gross matter. What is the effect of this pressure after it has left the sun and before it has again reached gross matter? It must have some effect on the æther, but the equations expressing that effect are wanting. If we tie ourselves down to a strict dynamical theory these equations appear, and we have to decide what is their meaning. In case I., on the present hypothesis, there is no doubt at all about the meaning. It can be expressed either by the equation

$$
\mathrm{V}\left(4 \pi \mathbf{D}^{\prime} \nabla^{\prime} \mathbf{E}^{\prime}+\mathbf{B}^{\prime} \nabla^{\prime} \mathbf{H}^{\prime}\right)=0, . . .
$$

or by saying that the Poynting intensity remains constant for every element of the æther. It follows also that there is no ponderomotive action and reaction between the æther and gross matter, which has an obvious bearing on the question of whether or not the æther can exert a pressure on gross matter.

Are these results consistent with an electromagnetic theory of light? I think they are not, but this is not on account of the most obvious apparent consequences. Take, for instance, the fact of the constancy of the Poynting intensity. From this we deduce that if any part of the æother were ever electrically at rest the actual displacement and magnetic force for that part would always be parallel, and if the displacement and magnetic force were for any part of the æther ever not parallel that part could never come electrically to rest.

8. These results are not necessarily inconsistent with a theory of light, for it must be remembered that the æther now contemplated is capable of indefinite expansion or condensation, and that whatever be the amount of this expansion or condensation its electrical constants remain absolutely unaffected. But by indefinite expansion the volume distribution of Poynting intensity is indefinitely diminished, and conversely for condensation. In fact, the æther is capable of 
moving, and in case of a forced irregular disturbance would move with velocities comparable with that of light. This will explain how we can on the present hypothesis account for the encroachment with sufficient rapidity of light waves on a previously dark region.

But there is a difficulty I have not been able to surmount. Eq. (14) is not linear in the electromagnetic vectors, and hence in general the superposition of two solutions of our equations does not give a third solution. I will show directly how a single plane wave which is sufficiently arbitrary to leave room for the explanation of polarized light \&c. can be borne by our æther, but I have failed in the endeavour to see what the effect on each other of two plane waves inclined to one another would be. It looks as if they must be altered in kind by their encounter, and this is contrary to several optical facts.

9. Without further preface I proceed to the consideration of case I. It may be remarked that a great part of the work below relating to this case is applicable to ordinary dielectrics, so that though I am inclined to reject the case, the work is not thrown away.

Our equations are now (5), (11), and (14). Equations (11) are for present purposes more conveniently written by eq. (23) $\$ 63$ of ' Electromag.'

$$
\left.\begin{array}{rl}
(4 \pi)^{-1} \nabla^{\prime} \mathbf{H}^{\prime} & =\partial \mathbf{D}^{\prime} / \partial t+V \nabla^{\prime} V \mathbf{D}^{\prime} \sigma^{\prime} \\
-\nabla^{\prime} \mathbf{E}^{\prime} & =\partial \mathbf{B}^{\prime} \partial t+\nabla \nabla^{\prime} \nabla \mathbf{B}^{\prime} \sigma^{\prime}
\end{array}\right\}, \quad .
$$

the $V$ being unnecessary before $\nabla^{\prime} \mathbf{H}^{\prime}$ and $\nabla^{\prime} \mathbf{E}^{\prime}$ when $K^{\prime}$ and $\mu^{\prime}$ are constant scalars, as we suppose to be the case for the æther. Putting now

$$
\begin{aligned}
& \mathbf{H}^{\prime} \nu \mu^{\prime}=\mathrm{H}_{0}, \quad \mathbf{E}^{\prime} \nu \mathrm{K}^{\prime}=\mathrm{E}_{0}, \quad v^{2} \mu^{\prime} \mathrm{K}^{\prime}=1, \quad \text {. } \\
& \left.\begin{array}{rl}
v \nabla^{\prime} \mathbf{H}_{0} & =\partial \mathbf{E}_{0} / \partial t+V \nabla^{\prime} \mathbf{V E}_{0} \sigma^{\prime} \\
-v \nabla^{\prime} \mathbf{E}_{0} & =\partial \mathbf{H}_{0} / \partial t+V \nabla^{\prime} \mathbf{V H}_{0} \sigma^{\prime}
\end{array}\right\} . . . .
\end{aligned}
$$

With this notation eq. (14) becomes

$$
\mathrm{V}\left(\mathbf{E}_{0} \nabla^{\prime} \mathbf{E}_{0}+\mathbf{H}_{0} \nabla^{\prime} \mathbf{H}_{0}\right)=0 . \quad \text {. . . . . }
$$

Notice that if $\sigma^{\prime}$ is zero equations (17) reduce to Maxwell's. Hence any solution of Maxwell's which also satisfies eq. (18), or, what amounts to the same, when $\sigma^{\prime}$ is zero any solution of Maxwell's which renders $\mathrm{VE}_{0} \mathbf{H}_{0}$ constant at every point is a solution also of our problem. We shall be led to such a solution below.

10. The only case $I$ have been able to discuss is a plane wave with $\mathbf{E}_{0}$ and $\mathbf{H}_{0}$ in the front. Suppose, then, $\mathbf{E}_{0}$ and $\mathbf{H}_{0}$ 
are functions of $z$ and $t$ only, which are perpendicular to $k$ $[i, j, k$ taken as usual with reference to the rectangular axes $x, y, z]$. Thus

$$
\nabla^{\prime}=k \partial / \partial z, \quad \cdot \quad \cdot \quad \cdot \quad \cdot \quad \cdot
$$

and therefore $\nabla^{\prime}$ is commutative with $k$. Put

$$
\mathbf{E}_{0}-k \mathbf{H}_{0}=2 \alpha, \quad \mathbf{E}_{0}+k \mathbf{H}_{0}=2 \beta, \quad \text {. . }
$$

which give

$$
\mathbf{E}_{0}=\alpha+\beta, \mathbf{H}_{0}=k(\alpha-\beta) . \quad . \quad . \quad \text {. }
$$

Equation (18) now gives

or

$$
\mathrm{S}(\alpha \partial \alpha / \partial z+\beta \partial \beta / \partial z)=0,
$$

$$
\alpha^{2}+\beta^{2}=-\mathrm{X}^{2},
$$

where $\mathrm{X}$ is a function of the time only.

From the constancy of the Poynting intensity we see that it is needless to consider a more general value of $\sigma^{\prime}$ than $u k$, where $u$ is a scalar function of $z$ and $t$ only. Substituting this value and multiplying the second of equations (17) by $k$ and adding it to and subtracting it from the first we obtain

$$
\begin{aligned}
& \partial \alpha / \partial t+\partial\{(u+v) \alpha\} / \partial z=0 . \\
& \partial \beta / \partial t+\partial\{(u-v) \beta \quad \partial z=0 .
\end{aligned}
$$

These give at once

$$
\begin{aligned}
& a=\partial \gamma / \partial z,(u+v) \alpha=-\partial \gamma / \partial t, \quad . \quad . \\
& \beta=\partial \delta / \partial z,(u-v) \beta=-\partial \delta / \partial t, \quad .
\end{aligned}
$$

where of course $\gamma, \delta$, like $\alpha, \beta$, are vector functions of $z$ and $t$ only, which are perpendicular to $k$.

Suppose $\gamma=\gamma_{1} i+\gamma_{2} j$, where $\gamma_{1}$ and $\gamma_{2}$ are scalars. Since $\partial \gamma / \partial z$ and $\partial \gamma / \partial t$ are parallel, it follows that

or

$$
\frac{\partial \gamma_{1}}{\partial z} / \frac{\partial \gamma_{1}}{\partial t}=\frac{\partial \gamma_{2}}{\partial z} / \frac{\partial \gamma_{2}}{\partial t}
$$

$$
\mathrm{J}\left(\begin{array}{cc}
\gamma_{1} \gamma_{2} \\
z & t
\end{array}\right)=0 . . . . . .
$$

Hence $\gamma_{1}$ and $\gamma_{2}$ and therefore $\gamma$ are functions of a single scalar function $\theta$ of $z$ and $t$. $\theta$ is to a certain extent arbitrary, since any function of $\theta$ will serve instead of $\theta$. Thus

$$
\gamma=\gamma(\theta), \text {. . . . . }
$$


where $\gamma(\theta)$ stands for any vector function of $\theta$ which is perpendicular to $k$. Similarly

$$
\delta=\delta(\phi),
$$

where $\phi$ and $\delta(\phi)$ are functions exactly similar to $\theta$ and $\gamma(\theta)$.

Equations (25) and (26) now give

$$
\begin{aligned}
& \partial \theta / \partial t+(u+v) \partial \theta / \partial z=0, \\
& \partial \phi / \partial t+(u-v) \partial \phi / \partial z=0 .
\end{aligned}
$$

These show that if a point move in the direction of $k$ faster by $v$ than the æther, $\theta$ will for it remain constant, and if a point move in the direction of $-k$ faster by $v$ than the æther, $\phi$ will for it remain constant. In other words, $\theta$ and $\phi$ are propagated relatively to the rther with the velocity $v$ in the direction of and that opposite to $k$.

11. We may drop the $\gamma$ and $\delta$ used above by putting

$$
\alpha=\alpha(\theta) . \partial \theta / \partial z, \beta=\beta(\phi) . \partial \phi / \partial z, .
$$

where $\alpha(\theta)$ and $\beta(\phi)$ are vector functions of $\theta$ and $\phi$ which are perpendicular to $k$. Equations (29), (30), and (31) are exactly equivalent to all the former equations involving $\alpha, \beta$, $\gamma$, and $\delta$.

On account of the arbitrariness of $\theta$ and $\phi$ they may be chosen so that $\partial \theta / \partial z$ and $\partial \phi / \partial z$ are each unity, or, again, they may be taken as the angles $\alpha$ and $\beta$ make with $i$.

The unknown $u$ may of course be eliminated from equations (29) and (30), leaving but one equation, but clearer ideas seem to be obtained by not effecting this elimination.

Let us now confine ourselves to propagations in one direction, and therefore put $\beta=0$. Take $\theta$ so that $\partial \theta / \partial z=1$, i.e. $\theta=z-\mathrm{Y}^{\prime}$, where $\mathrm{Y}^{\prime}$ is a function of $t$ only. Substituting this value of $\theta$ in equation (29),

$$
u=\partial \mathrm{Y}^{\prime} / \partial t-v \text {. }
$$

Hence if the rether be oscillating about a mean position

$$
\partial \mathrm{Y}^{\prime} / \partial t=v+\mathrm{Y}^{\prime \prime}
$$

where $Y^{\prime \prime}$ is a function of $t$ oscillating about the value zero. It follows that $\mathrm{Y}^{\prime}=v t+\mathrm{Y}$ or

$$
\theta=z-v t-\mathrm{Y}, \cdot \ldots \cdot \cdot \cdot
$$

where $\mathrm{Y}$ is an oscillatory function of $t$, whose average value is zero.

The solution so far is applicable to an isotropic dielectric, for which $K^{\prime}$ and $\mu^{\prime}$ are independent of strain. In this aspect it will be returned to later. 
12. For the æther at present under consideration we see by equation (22) that $\alpha^{2}$ and therefore $\tau \alpha$ is a function of the time only. But it is a function (in the case of waves) of $t$ and $z$, i. e. it is a constant.

Let $\mathrm{Y}$ of equation (32) be zero. Suppose at any instant lines are drawn from all points of the axis of $z$ equal and parallel to the values of $\alpha$ there. Their extremities will lie on a right cylinder with $z$ for axis, and the curve they form will be a complete representation of the wave. Figures 1, 2, 3,4 thus represent different types of waves that can be borne

Fig. 1.

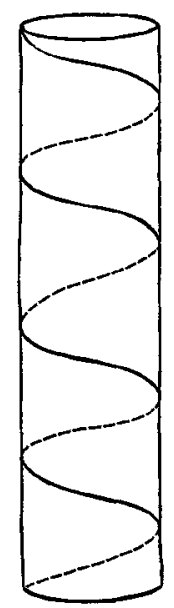

Fig. 2.

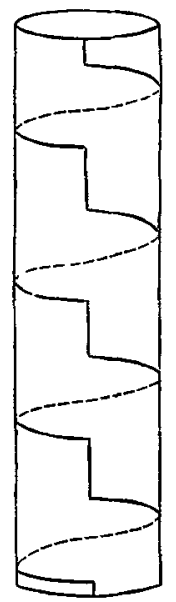

Fig. 3.

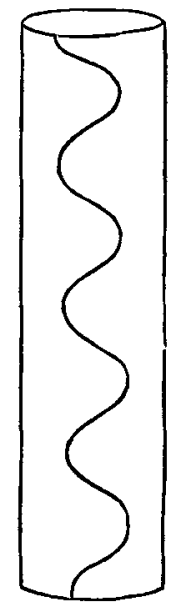

Fig. 4.

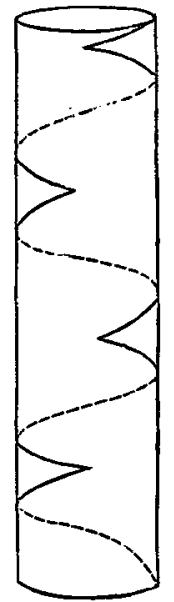

by our æther. Fig. 1 is of course a circularly polarized wave. Fig 2 is a wave partly plane-polarized and partly circularly polarized, i.e. elliptically polarized. Fig. 3 would probably appear to us as a plane-polarized, and fig. 4 as ordinary unpolarized light.

All such waves are particular ones of Maxwell's. Now Maxwell asserts that when such waves are incident on gross matter the æether exerts a pressure on that matter. But as we have seen, on the present theory all ponderomotive forces consist of actions and reactions between different parts of gross matter and never between gross matter and æther, and yet for the present case our stress is exactly the same as Maxwell's. The explanation of the apparent discrepancy is contained in $\$ 75$ of 'Electromag.' If we find that a certain stress suffices to explain certain experimental facts, there are an infinite number of other stresses that will also do so. We 
do not therefore conclude that on the present theory this pressure of Maxwell's would not exist, but we do conclude that it does not exist for eight minutes in the æther between the sun and earth, that in fact at the instant when the earth experiences the pressure the sun or some other body experiences the reaction. This is, of course, because in the present theory the conditions of equilibrium are always satisfied by the other.

13. Take $\theta$ as the angle that $a$ makes with $i$. The solution of $\$ 12$ with this meaning of $\theta$ is

$$
\alpha=\mathrm{A}(i \cos \theta+j \sin \theta), . . . .
$$

where $A$ is a constant and $\theta$ is any function of $z-v t$, for instance $2 \pi(z-v t) / \lambda$. I will now show that it is impossible for two waves of this type inclined to one another to coexist.

Fig. 5.

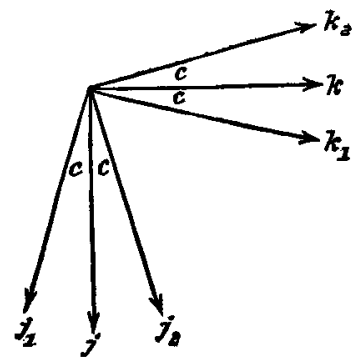

Let $i$ be drawn perpendicular to the plane of the paper upwards. Let $j, k, j_{1}, k_{1}, j_{2}, k_{2}$ be related as shown in fig. 5 . It is required to find whether with $\sigma^{1}$ zero the following equations can be satisfied :-

$$
\left.\begin{array}{l}
\mathbf{E}_{0}=\mathbf{E}_{01}+\mathbf{E}_{02}, \mathbf{H}_{0}=\mathbf{H}_{01}+\mathbf{H}_{02}, \\
\alpha_{1}=\mathbf{A}_{1}\left(i \cos \theta_{1}+j_{1} \sin \theta_{1}\right), \mathbf{E}_{01}=\alpha_{1}, \mathbf{H}_{01}=k_{1} \alpha_{1} \\
\boldsymbol{\alpha}_{2}=\mathbf{A}_{2}\left(i \cos \theta_{2}+j_{2} \sin \theta_{2}\right), \mathbf{E}_{02}=\alpha_{2}, \mathbf{H}_{02}=k_{2} \alpha_{2}
\end{array}\right\},
$$

where $A_{1}$ and $A_{2}$ are constants, and $\theta_{1}$ and $\theta_{2}$ are functions of $z_{1}$ and $t$ and of $z_{2}$ and $t$ respectively.

Since $\sigma^{\prime}$ is zero these values of $E_{0}$ and $\mathbf{H}_{0}$ satisfy equation (17), and we know that in this case equation (18) is equivalent to the equation $\nabla E_{0} H_{0}=a$ constant throughout time and therefore throughout space. We have seen that $V \mathrm{~F}_{01} \mathrm{H}_{01}$ and $\nabla E_{02} H_{02}$ are such constants, so that it only remains to make if possible $\mathrm{V}\left(\mathrm{E}_{01} \mathbf{H}_{02}+\mathbf{E}_{03} \mathbf{H}_{01}\right)$ a constant. Now 


$$
\begin{aligned}
\nabla\left(\mathbf{E}_{01} \mathbf{H}_{02}+\mathbf{E}_{02} \mathbf{H}_{01}\right)=\mathrm{V}\left(\alpha_{1} k_{2} \alpha_{2}+\alpha_{2} k_{1} \alpha_{1}\right) & \\
= & \mathrm{A}_{1} \mathrm{~A}_{2} \mathrm{~V}\left\{\left(i \cos \theta_{1}+j_{1} \sin \theta_{1}\right)\left(-i \sin \theta_{2}+j_{2} \cos \theta_{2}\right)+\left(i \cos \theta_{2}\right.\right. \\
& \left.\left.\quad+j_{2} \sin \theta_{2}\right)\left(-i \sin \theta_{1}+j_{1} \cos \theta_{1}\right)\right\} \\
= & \mathrm{A}_{1} \mathrm{~A}_{2}\left\{\left(k_{1}+k_{2}\right) \cos \left(\theta_{1}-\theta_{2}\right)+\mathrm{V} j_{1} j_{2} \sin \left(\theta_{1}-\theta_{2}\right)\right\} \\
= & \mathrm{A}_{1} \mathrm{~A}_{2}\left\{2 k \cos \mathrm{C} \cos \left(\theta_{1}-\theta_{2}\right)+i \sin 2 \mathrm{C} \sin \left(\theta_{1}-\theta_{2}\right)\right\},
\end{aligned}
$$

where $2 \mathrm{C}$, as indicated in fig. 5 , is the angle between $j_{1}$ and $j_{2}$ and between $k_{1}$ and $k_{2}$. Hence when neither $\cos C$ nor $\sin \mathrm{C}$ is zero, $i$.e. when the waves are not parallel, we must have both $\cos \left(\theta_{1}-\theta_{2}\right)$ and $\sin \left(\theta_{1}-\theta_{2}\right)$ constant throughout space and time, i.e. $\theta_{1}-\theta_{2}$ must be constant. But since $\theta_{1}$ involves $z_{1}$ and $\theta_{2}$ involves $z_{2}$, this is impossible. Hence two such waves cannot coexist.

14. This shows that if a wave incident on a reflecting surface be of the present type, it is if in free æther altered in type by the reflected wave.

Even this, however, is not completely condemnatory of the present hypothesis ; for

(1) Although such waves will be altered in type we have not shown in what manner, and $I$ am at present unable to do so. They may be only so altered that the alteration does not affect our senses. This, however, I consider extremely improbable.

(2) We have no experimental evidence that in free other such alteration does not occur. In our most perfect vacua the density is such that if they could be assumed continuous material plenums, Maxwell's ignoration of the equilibrium equation would be justifiable. Though we have every reason to believe that they are not such plenums, yet owing to our ignorance of the effect on the zther of the proximity of material atoms, I think we are justified in so regarding them for present purposes. If this assumption be made, Maxwell's theory of light is applicable to all terrestrial experiments. It would still remain on the present hypothesis to be proved that the apparent positions and spectra of the hearenly bodies are not appreciably altered by the interferences just considered. This would seem by no means so difficult as the task just now proposed.

But there is another grave difficulty. We have no guarantee that the velocity of light in space will be practically a constant. If, for instance, there were in the solar system a circulation of the ether whose velocity was comparable with that of light, the apparent positions of the sun and planets would be largely affected.

Phil. Mag. S. 5. Vol. 36. No. 219. Aug. 1893. 
15. However this be, our ideas would be greatly simplified if Maxwell's ignoration of the equilibrium equation could be justified by some suitable modification of the fundamental assumptions.

Let us now assume that the æother has a material density sufficiently large to render $\sigma^{\prime}$ small compared with the velocity of light. This is case II. of $\$ 6$ above. In this case Maxwell's ignoration will be justified.

To fix the ideas let us suppose that a plane-polarized wave with the energy of strong sun-light causes the maximum æther velocity to be $b$ times that of light where $b$ is small. $\sigma^{\prime}$ may then be regarded as a small quantity in equations (15) and (17), so that equations (15) reduce to Maxwell's.

Since we now suppose the velocity to be small and oscillatory, the present and standard positions may be assumed to coincide, and we have

$$
\begin{aligned}
& \mathrm{E} \nu \mathrm{K}=\mathrm{A} i \cos \{2 \pi(z-v t) / a\}=\mathrm{A} i \cos \theta, \\
& \mathbf{H} \nu \mu=\mathrm{A} j \cos \theta,
\end{aligned}
$$

for the wave we contemplate. The energy $\lambda$ per unit volume is given by

$$
\lambda=-\left(\mathrm{KH}^{2}+\mu \mathrm{H}^{2}\right) / 8 \pi=\mathrm{A}^{2} \cos ^{2} \theta / 4 \pi .
$$

Hence the average value $\bar{\lambda}$ of $\lambda$ is

$$
\bar{\lambda}=A^{2} / 8 \pi=4 \cdot 225 \times 10^{-5}
$$

in ergs per cub. centim., according to the data given in $\S 793$ of Maxwell's 'Electricity and Magnetism.'

The velocity of the æther may be calculated from Maxwell's stress, or written down at once from equation (12) above. For in the present case $W, P$, and $P_{0}$ are zero, and $\sigma^{\prime 2}$ may be neglected. Thus

$$
\begin{aligned}
\mathrm{D} \dot{\sigma} & =d \mathrm{VDB} / d t=(4 \pi v)^{-1} \mathrm{~A}^{2} k d\left(\cos ^{2} \theta\right) / d t \\
& =v^{-1} \bar{\lambda} k d(1+\cos 2 \theta) / d t .
\end{aligned}
$$

Since $\sigma$ is to vary about a mean value

Thus

$$
\mathrm{D} \sigma=v^{-1} \bar{\lambda} k \cos 2 \theta \text {. }
$$

$$
b=\sigma_{m} / k v=\bar{\lambda} / v^{2} \mathrm{D},
$$

where $\sigma_{m}$ is the maximum value of $\sigma$. Thus approximately

$$
6 \mathrm{D}=5 \times 10^{-26} \text {. }
$$

The lower limit obtained by Lord Kelvin for the density of æether ('Math. and Phys. Papers,' ii. 32) is

$$
\mathrm{D}=10^{-22}
$$

approximately. In this case $b=1 / 2000$. There seems then 
no difficulty on the mere ground of requisite density in explaining the justifiability of ignoring the equation of equilibrium. And we see that in all material transparent bodies we are fully warranted.

I have not, however, been able to satisfy myself that in the present case the æother density is stable, i.e. that it will not gather itself up round nuclei. If it did so, its density not in the neighbourhood of these nuclei might be too small to enable us to ignore the equation of equilibrium.

16. Case III. seems the most satisfactory one. Suppose the rther absolutely rigid and motionless except in a thin skin coating every atom of matter. Suppose in this skin it has exactly the same freedom as in case I. This is equivalent to supposing that in the other the terms of $l$ depending only on $\Psi$ vary infinitely rapidly with all variations of $\Psi$ except in the skin, where they are zero. In this case the atoms of matter will be unimpeded in their motion through the æether, the equation of equilibrium can be rigorously ignored for the æther, the electrical equations for the æther reduce to Maxwell's form, and the investigation in $\$ 64$ of 'Electromag.' concerning convection currents is made satisfactory.

These assumptions sound highly artificial, so it is well to remark that the mathematical results of a theory apparently different in fundamental assumptions are identical. This theory may be stated in the following different forms :-

(1) The æther is immovable and freely penetrates all atoms offering no resistance to their motion.

(2) 'The theory of 'Electromag.' is true of matter, but Maxwell's theory is true of free æether.

(3) For matter the theory of 'Electramag.' is true. For space where there is no matter we have in place of the fundamental assumptions of $\$ \S 23$ to 31 the following :-For such space the undashed letters have no meaning. The Lagrangian function is the volume-integral of

$$
\begin{gathered}
2 \pi \mathrm{K}_{0}^{-1} \mathbf{D}^{\prime 2}-\mu_{0} \mathbf{H}^{\prime 2} / 8 \pi . \\
\mathbf{k}^{\prime}=0 . \quad \mathrm{S} \nabla^{\prime} \mathbf{D}^{\prime}=0 . \quad \mathrm{V} \nabla^{\prime} \mathbf{H}=4 \pi \mathbf{C}^{\prime}=4 \pi \partial \mathbf{D}^{\prime} / \partial t .
\end{gathered}
$$

In addition certain assumptions must be made with regard to the bounding surface of matter. They are most readily expressed by saying that for a thin skin bounding the matter' the assumptions of 'Electromag.' are true, and that for this region the Lagrangian function per unit volume of the standard position is $2 \pi K_{0}^{-1} D^{2}-\mu_{0} H^{2} / 8 \pi$, the outer surface of the skin being motionless and the inner surface moving continuously with the matter.

I have given these three forms to indicate that the theory 02 
is not so artificial as it at first sight appears. For mathematical purposes the first form of the theory is the most convenient.

17. Applying the solution of $\S 11$ to a moving dielectric (constant scalar permeability and capacity) we see that the velocity of light ought on the present theory to be altered by the full value of the velocity of the medium. Thus the theory requires some modification to explain Michelson's results, \&c. The last section at once suggests a possible form of such modification, viz. that the theory of 'Electromag.' is true for the interior of material atoms but not for the æther in their immediate vicinity. In 'Electromag.' the individual atoms of matter were not taken into account. To meet the present theory on the lines of 'Electromag.' it would be necessary to make some modification in the fundamental assumptions.

Hobart, April 8, 1893.

XVII. Further Data on Colour-Blindness.-No. IV. By Dr. William Pole, F.R.S.*

HeRING's Explanation of the defect.

$\mathbf{E}^{\mathrm{v}}$ VERYBODY who has had to do with colour-blindness has heard of the connexion with it of the eminent physiologist, Professor Ewald Hering, of Prague ; but it is remarkable how little is known here of his actual writings. Between 1872 and 1874 he laid before the Imperial Academy of Sciences of Vienna six communications on the vision of light and colours, which were afterwards published separately $\dagger$, and have how become classical in the literature of the subject. In 1879 I published in 'Nature' a set of articles giving an abstract of the work, and this abstract has formed, I believe, the only direct account in the English language of Hering's labours in the department of colourvision, although his writings thereon have been legion.

This work, however, does not refer to colour-blindness, except in a few lines promising a future special treatment of it. The promise was fulfilled in 1880 by an Essay entitled "Zur Erklärung der Farbenblindheit, aus der Theorie der Gegenfarben," published at Prague in a scientific serial called 'Lotos.' Some reprints were circulated, of which I was fortunate enough to get one; but it is very scarce, and is probably little known in England except to the for who interest themselves specially in these foreign scientific views.

* Communicated by the Author.

† Zur Lehre vom Lichtsinne. Zweiter Abdruck. Wien, 1878. 